\title{
Design and Operation of a Calorimeter for Advanced Multilayer Insulation Testing
}

\author{
David J. Chato*, Wesley L. Johnson, and Neil T. Van Dresar (ret.) \\ NASA Glenn Research Center, Cleveland, OH, 44135
}

\begin{abstract}
A calorimeter has been constructed to accurately measure insulation performance with a nominal $90 \mathrm{~K}$ outer boundary and a $20 \mathrm{~K}$ inner boundary. Unique features of this design include use of mechanical cryocoolers instead of cryogens and measurement of the heat load with a calibrated heat conduction rod. The calorimeter is operational and has completed its first test series. The initial test series was designed to look for differences in performance between a single layer of aluminum foil and a sheet of double aluminized Mylar (DAM). Although it has been speculated that the aluminum foil would perform better, since the aluminum coating on the Mylar might not be thick enough to stop the transmission of long wave length infrared radiation, our testing showed a higher heat load for the aluminum foil than the DAM. The aluminum foil showed a heat load of $132 \mathrm{~mW}$ at an $87 \mathrm{~K}$ outer temperature and $152 \mathrm{~mW}$ at a $107 \mathrm{~K}$ outer temperature, whereas the DAM showed a heat load of $66 \mathrm{~mW}$ at an $88 \mathrm{~K}$ outer temperature and $81 \mathrm{~mW}$ at $108 \mathrm{~K}$.
\end{abstract}

\section{Introduction}

Multilayer insulation (MLI) is considered the state of the art insulation for cryogenic propellant tanks in the space environment. MLI consists of multiple layers of metalized films separated by low conductivity spacers. The layers (metalized films) act as radiation shields and the spacers minimize thermal shorting between layers. Typically, MLI thermal performance data is obtained from tests of MLI specimens (coupons) conducted with calorimeters having warm and cold boundary temperatures representative of the conditions a MLI system would be exposed to on a spacecraft. Warm boundary temperatures are typically 200 to $300 \mathrm{~K}$, representing exposure to the Sun or Earth. Cold boundary temperatures are less than $100 \mathrm{~K}$, representing the wall temperature of cryogenic propellant storage tanks. There is a gap in existing test capability at cold boundaries of $20 \mathrm{~K}$. Existing test facilities at Kennedy Space Center have been demonstrated that have cold boundary temperatures of $77 \mathrm{~K}$ using liquid nitrogen. [1] [2] Similarly, a calorimeter has been developed at Florida State University that has capability at $20 \mathrm{~K}$ using cryocoolers as the heat sink. [3] Testing performed for the Cryogenic Propellant Storage and Transfer (CPST) and Self Supporting MultiLayer Insulation (SS-MLI) projects measured higher heat loads than expected, the cause of which, could not be readily resolved. [4] Another calorimeter using a helium gas temperature difference is currently in the final stages of check out at the Technical University of Dresden, and while it also claims to be capable of cold boundary temperatures in the $20 \mathrm{~K}$ range, it has yet to be demonstrated. [5] With this state of the calorimetry for low temperature testing, it was decided to build a calorimeter at the NASA Glenn Creek Road Cryogenic Complex (CRCC) for use at the Cell-7 test facility.

\section{Basic Design of Calorimeter}

The calorimeter was constructed to measure the performance of MLI using cryocoolers rather than cryogens. The Key advantages of this choice of calorimeter include: not needing to use and top-off with cryogens, less safety restrictions on unattended operation and location of test rig since volatile cryogens are not present, and a wider range of boundary temperatures. The intended operation of the system was for boundary temperatures of $20 \mathrm{~K}$ on the cold side and $90 \mathrm{~K}$ on the warm side. This was intended to investigate long reported issues with MLI at low boundary temperatures $[4,7,8,9]$. A conceptual drawing of the calorimeter is shown in figure 1 . The calorimeter consists of cold and warm surfaces which are a pair of nested cylinders with flat ends inside a cylindrical vacuum vessel. Figure 2 shows a view of the cylinders installed in the vacuum vessel. The cold (inner) cylinder has guarded top and bottom ends to minimize the effects of heat transfer at the ends of the test section. The guards are thermally separated from

\footnotetext{
* Associate Fellow, American Institute of Aeronautics and Astronautics
} 
the test section by a 1/4" gap. They are structurally connected with thermally insulating G10 tabs. The inside of the warm (outer) cylinder and outside of the cold cylinder are painted with Aeroglaze Z306 (room temperature emissivity 0.90 , solar absorptivity $>0.95$ ) to provide a black body surface for the insulation to view. Both cylinders are shown in figure 3. The warm cylinder, test section of the cold cylinder, and a pair of guarded ends on the cold cylinder are each cooled by a cryocooler ( 3 total, the two guards are controlled by a separate cryocooler than the test section). The working portion of the cryocoolers are shown installed on the vacuum lid in figure 4. Each cryocooler is paired with a compressor (not shown) that provides high pressure gas to drive the cryocooler. Each cryocooler-cooled subsystem is fitted with a trim heater to adjust the temperature of that subsystem. The MLI test specimen is wrapped around the sidewall of the cold cylinder. The portion of the heat flow from the warm cylinder through the MLI to the cold cylinder's test section must flow through the test section wall to a conductive plate (hub), then through a calibrated conduction rod (CCR), and last through a thermal strap to the cryocooler cold head similar to the configuration in [4]. A representative rod (one of a set six for different heat loads) is shown in figure 5.At steady state conditions, the heat flow through the MLI covering the test section is equal to the heat flow through the CCR. The thermal conductivity and cross sectional area of each CCR were chosen to provide an optimal temperature drop in each respective CCR. The heat flow through the CCR is calibrated as a function of the temperature readings at two locations along the CCR.

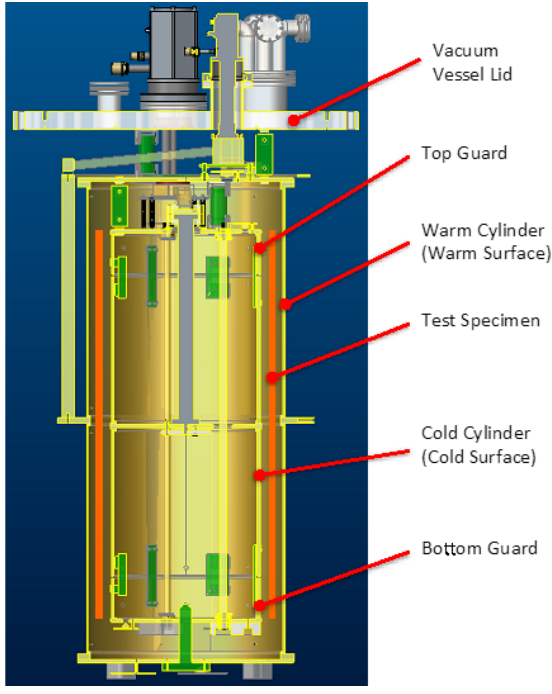

Figure 1 - Calorimeter Concept Drawing

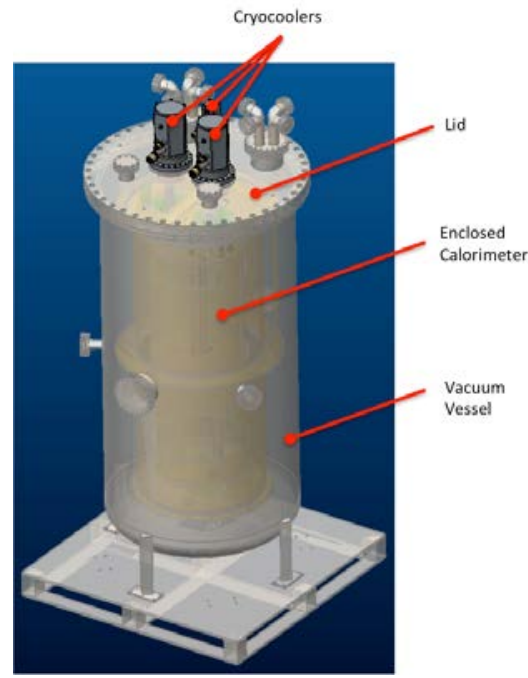

Figure 2 - Vacuum Vessel
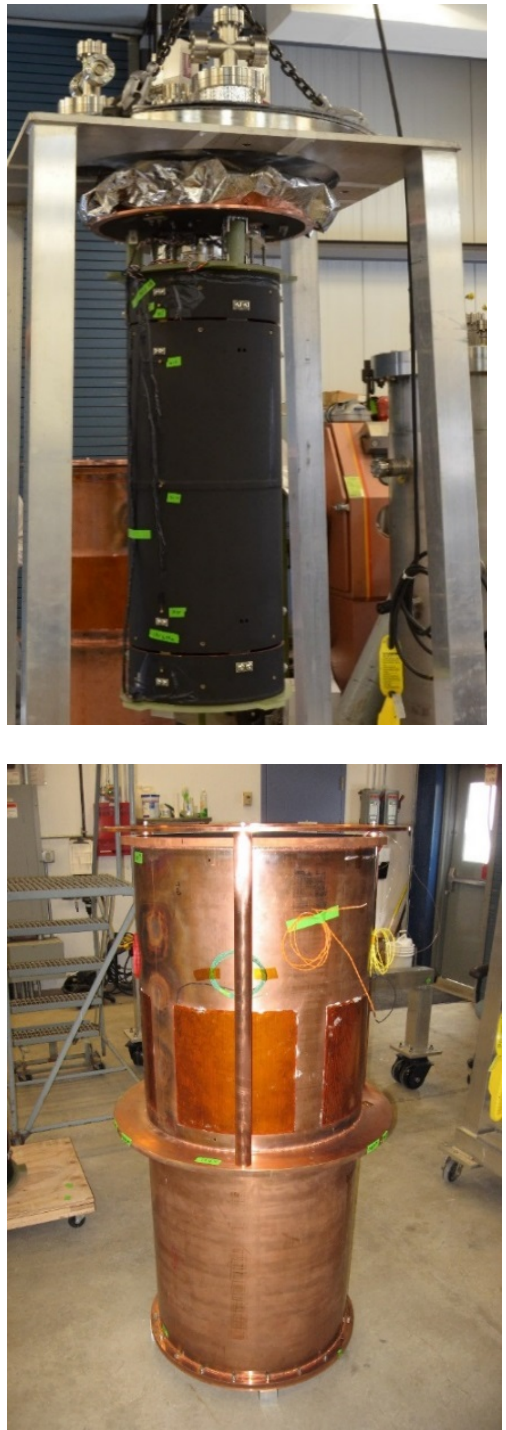

Figure 3 Inner and Outer Cylinders in preparation for test 


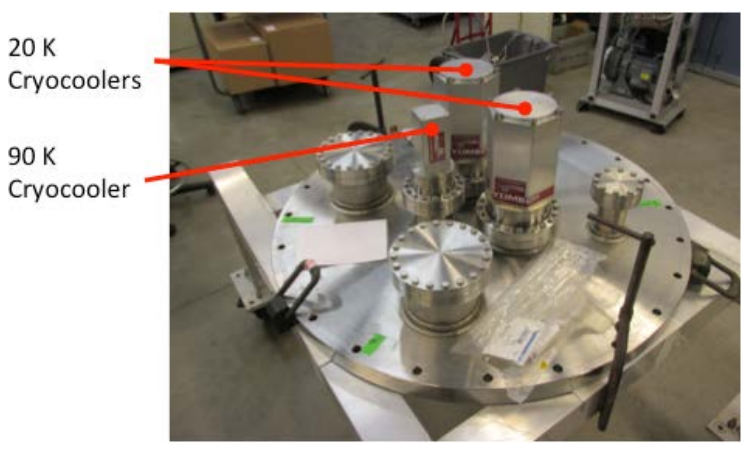

Figure 4 - Cryocoolers Mounted on Vacuum Vessel Lid

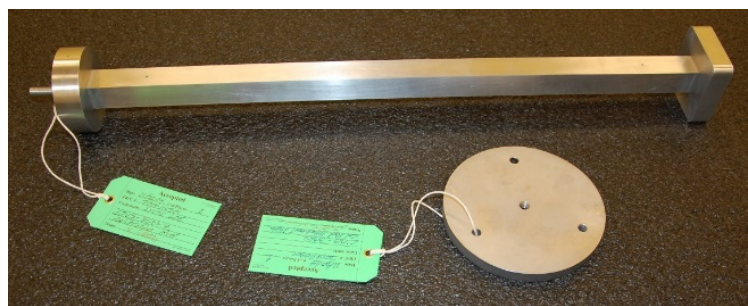

Figure 5 - Calibrated Conduction Rod (Dash 4) and Adapter Plate

Checkout testing was completed where the system heater control constants for the Proportional Integral Differential (PID) controllers were determined and systems operation demonstrated for $90 \mathrm{~K}$ temperatures on the outer cylinder and $20 \mathrm{~K}$ temperatures on the inner cylinder and guards.

\section{Calibration}

\section{A. Calibration Rig Description}

Calibration is performed in the calibration rig, shown in Figure 6. The rig consists of replacing the inner and outer cylinders with a smaller calibration cylinder and two heaters. The calibration cylinder surrounds the CCR being calibrated, provides a nearly isothermal environmental temperature for the CCR and effectively isolates the CCR from unwanted heat inputs. The calibration cylinder is connected to the $20 \mathrm{~K}$ cryocooler cold head via a thermal strap. The upper end of the CCR being calibrated is attached to the top of the calibration cylinder and is also cooled by the same cryocooler.

One heater (calibration heater) is installed in a hole bored into the lower end of the CCR. This heater supplies a measured power (or heat) input at the bottom of the CCR. The electrical power dissipated from the heater then flows thru the CCR and exits at its cold upper end. The other heater is installed in the top of the CCR. This heater is used to control the temperature of the calibration cylinder and the cold end of the CCR. By adjusting the power inputs to the two heaters the various calibration points $\left(\Delta T\right.$ and $T_{\text {ave }}$ ) can be obtained.

The calibration rig is placed inside the vacuum vessel. The top of the warm cylinder remains attached to the vacuum vessel lid and the warm cylinder thermal strap is left in place, but otherwise the warm cylinder is removed from the vacuum vessel. The cold cylinder is also removed from the vacuum vessel during use of the calibration rig. The calibration rig is suspended from the warm cylinder top by G-10 cylinders.

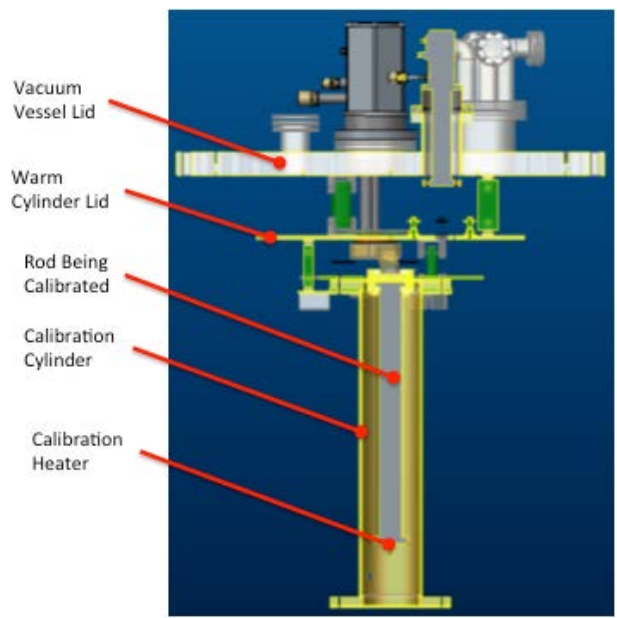

Figure 6 - Calibration Rig

American Institute of Aeronautics and Astronautics 
For each calibration point, the system is allowed to reach steady state, then the input power (voltage and current) is recorded, along with the temperature measured by each of the 4 temperature sensors on the CCR. Steady state requirements for calibration are specified as:

1. Steady conditions are required over a several hour period.

2. Calibration power as calculated from the voltage and current readings $\left(Q_{\text {cal }}=\right.$ Voltage $\times$ Current $)$ is constant to within \pm 1 percent.

3. All temperature readings on the CCR are constant to within \pm 1 percent.

4. The average temperature of the calibration cylinder (based on sidewall, top and bottom) is constant to within \pm 1 percent.

The calibration procedure involves obtaining steady state calibration data for a minimum of eight calibration points. The points should span the expected temperature operating range planned for the CCR being calibrated. The calibration process has been automated such that the entire calibration process occurs unattended.

The proposed form of the calibration equation is:

$$
\dot{Q}=a T_{a v g}^{2}+b T_{a v e}+c T_{a v e} \Delta T+d(\Delta T)^{2}+e \Delta T+f
$$

The coefficients $a-f$ will be fitted for each of the six pairs of temperature sensor combinations: $(1,2),(1,3),(1,4)$, $(2,3),(2,4)$ and $(3,4)$.

\section{B. Calibration of the Conduction Rod}

After initial checkout testing the conduction rod which serves as the main heat flow measurement system was calibrated. The software running the testing was designed to get the system to steady state and then run about 3.6 hours of steady state data for each point. By starting at the lowest temperatures, and slowly increasing the cold side temperature, time was able to be minimized with the first 10 data points collected in 48 hours. Three more data points were gathered after the initial data set was analyzed to get one point that was missed in the initial programming and also to repeat two tests to get a feel for system repeatability. It was immediately noticed that there was sometimes a significant difference between the measured heater power and the predicted rod heat flow. There is a clear linear relationship between this difference and the size of the temperature difference at the measure points. This relationship is illustrated in figure 7. Several options have been discussed as to the reason of the offset, including stray radiation and various conduction losses, with the current leading culprit thought to be conduction loads via the silicon diode wires and heater wires. A thermal model of the calibration system is currently in the process of being developed in hopes of providing a better answer. However, since there is one-to-one correspondence between heat flow and the rod temperature profile, heat flow measurements can still be made.

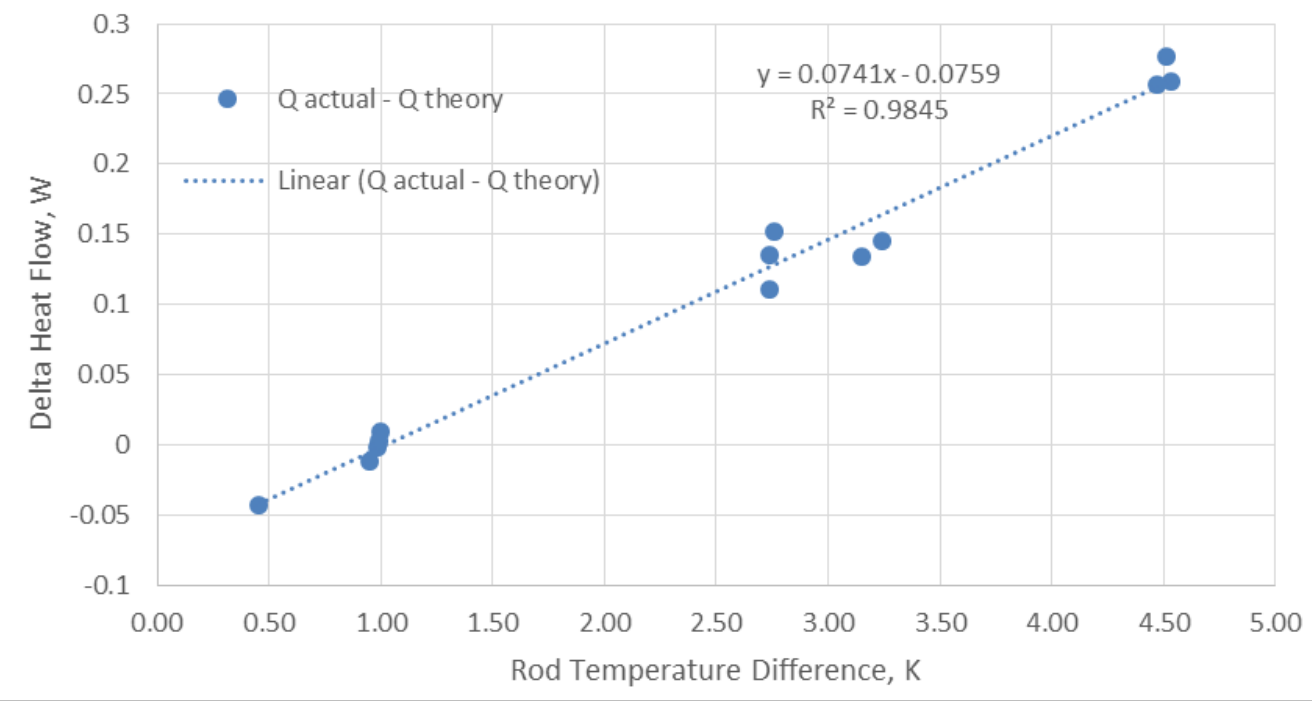

Figure 7 Difference between predicted and actual heat flow plotted as a function of rod temperature differential.

American Institute of Aeronautics and Astronautics 


\section{Testing of Single layers}

Once the conduction rod was calibrated, testing was begun to study the transmissive behavior of double aluminized mylar between $90 \mathrm{~K}$ and 20K. Single layers of both aluminum foil and double aluminized mylar were tested for thermal performance. By measuring the thermal performance of just one reflective layer it gave the opportunity to more easily recognize any transmittance as a degradation in performance. At 7 micrometers thick, the aluminum foil is nearly two orders of magnitude thicker than the aluminum layers on the aluminized Mylar. As such, there would be no transmission through the foil and any significant transmission would appear as degradation in thermal performance (increased heat load) through the double aluminized Mylar. Both the aluminized Mylar and the aluminum foil were attached to the top and bottom edge guards to eliminate direct thermal contact with the cold test section surface. Figure 8 shows the DAM coupon installed on the calorimeter. Room temperature emissivity was measured for both coupons prior to installation on the calorimeter in accordance with ASTM E-408 [6]. Table 1 shows the specified materials and expected test conditions.

Table 1: Test Matrix for Calorimeter Testing

\begin{tabular}{|c|c|c|c|c|c|c|}
\hline & Test Specimen & $\begin{array}{c}\text { Aluminum } \\
\text { Thickness, } \boldsymbol{\mu m}\end{array}$ & $\begin{array}{c}\text { Warm Boundary } \\
\text { Temperature, } \mathrm{K}\end{array}$ & $\begin{array}{c}\text { Cold Boundary } \\
\text { Temperature, } \mathbf{K}\end{array}$ & $\begin{array}{c}\text { Vacuum } \\
\text { Level, torr }\end{array}$ & $\begin{array}{c}\text { Room } \\
\text { Temp } \boldsymbol{\varepsilon}\end{array}$ \\
\hline Test 1 & $\begin{array}{c}\text { Aluminized } \\
\text { Mylar }\end{array}$ & $\begin{array}{c}0.10 \text { (on each } \\
\text { side) }\end{array}$ & 90 & 20 & $10^{-6}$ & $\begin{array}{c}0.13+/- \\
0.003\end{array}$ \\
\hline Test 2 & Aluminum Foil & 7.2 & 90 & 20 & $10^{-6}$ & $\begin{array}{c}0.046+/- \\
0.02\end{array}$ \\
\hline
\end{tabular}

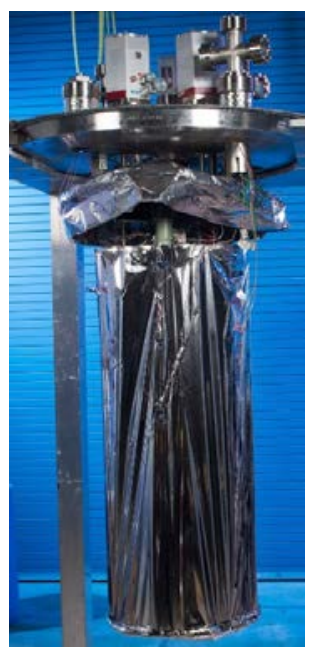

Figure 8 Double aluminized Mylar test coupon installed on the inner cylinder of the calorimeter.

\section{Results of Transmissivity Test}

Testing was performed using the calorimeter on two different materials at identical boundary conditions. In an extension of the test matrix, they were tested at two different warm boundary temperatures in an attempt to detect variation. The as tested performance is shown in Table 2. SD 31 is a temperature sensor placed 2.25" from where the cryocooler connects to the rod. SD 34 is 20" down the rod from SD31. Note that since our inner cylinder is held at 20 $\mathrm{K}$ the cryocooler end must be cooler to remove heat, hence SD31 drops below 20K. Sample test plots of system temperatures (warm boundary and cold boundary) are shown in Figures 9 and 10.

Table 2: Calorimeter raw test results.

\begin{tabular}{|c|c|c|c|c|c|c|}
\hline Test Coupon & $\begin{array}{l}\text { Vacuum } \\
\text { Pressure } \\
\text { (Torr) }\end{array}$ & $\begin{array}{l}\text { Warm Boundary } \\
(\mathbf{K})\end{array}$ & $\begin{array}{l}\text { Cold Boundary } \\
(\mathrm{K})\end{array}$ & $\begin{array}{l}\text { SD-31 } \\
(\mathrm{K})\end{array}$ & $\begin{array}{l}\text { SD-34 } \\
(\mathrm{K})\end{array}$ & $\begin{array}{l}\text { Calculated Heat } \\
\text { Load (mW) }\end{array}$ \\
\hline Aluminum Foil & $2.2 \times 10^{-8}$ & 87.1 & 20.1 & 16.77 & 19.80 & 281 \\
\hline Aluminum Foil & $2.2 \times 10^{-8}$ & 107.2 & 20.4 & 16.80 & 20.07 & 306 \\
\hline Aluminized Mylar & $1.8 \times 10^{-8}$ & 87.8 & 19.9 & 18.07 & 20.04 & 190 \\
\hline Aluminized Mylar & $1.2 \times 10^{-8}$ & 107.8 & 20.2 & 18.08 & 20.21 & 207 \\
\hline
\end{tabular}


After removing the conduction from the temperature sensors, the heat fluxes are shown in Table 3. As noted from the Calibration section, these are preliminary and subject to change upon completion of the thermal model.

Table 3: Calorimeter refined test results.

\begin{tabular}{|c|c|c|c|c|}
\hline Test Coupon & $\begin{array}{l}\text { Warm } \\
\text { Boundary (K) }\end{array}$ & Qnet (mW) & $\begin{array}{ll}\begin{array}{l}\text { Heat } \\
\left(\mathrm{mW} / \mathrm{m}^{2}\right)\end{array} & \text { Flux }\end{array}$ & $\begin{array}{l}\text { Effective } \\
\text { emissivity }\end{array}$ \\
\hline Aluminum Foil & 87.1 & 132 & 93 & 0.028 \\
\hline Aluminum Foil & 107.2 & 152 & 107 & 0.014 \\
\hline Aluminized Mylar & 87.8 & 66 & 46 & 0.014 \\
\hline Aluminized Mylar & 107.8 & 81 & 57 & 0.007 \\
\hline
\end{tabular}

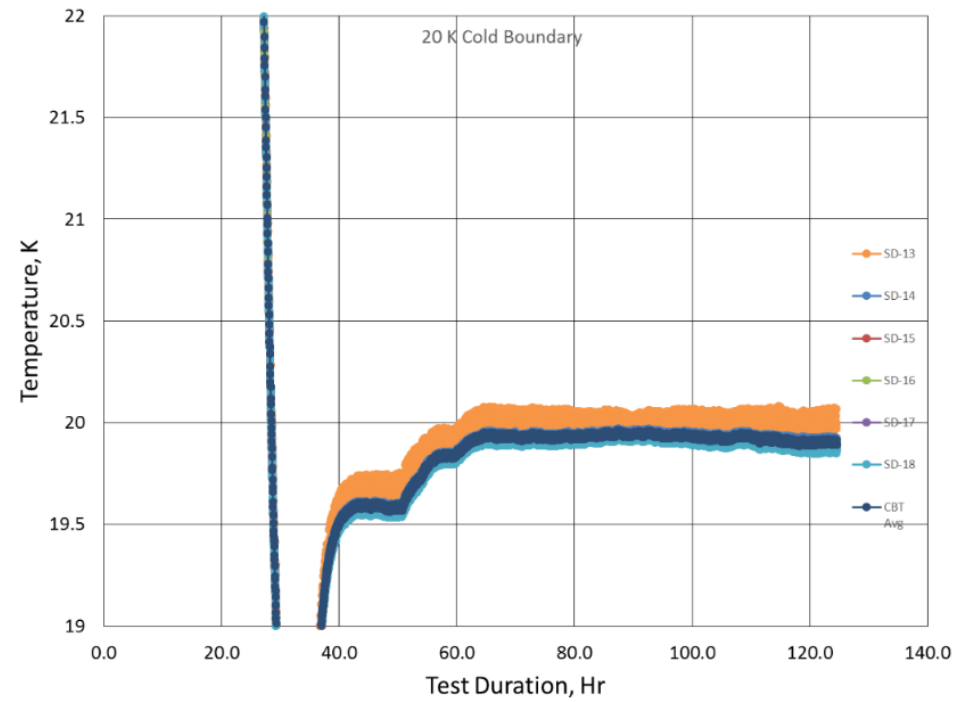

Figure 9: Cold Boundary Temperatures for double aluminized Mylar testing.

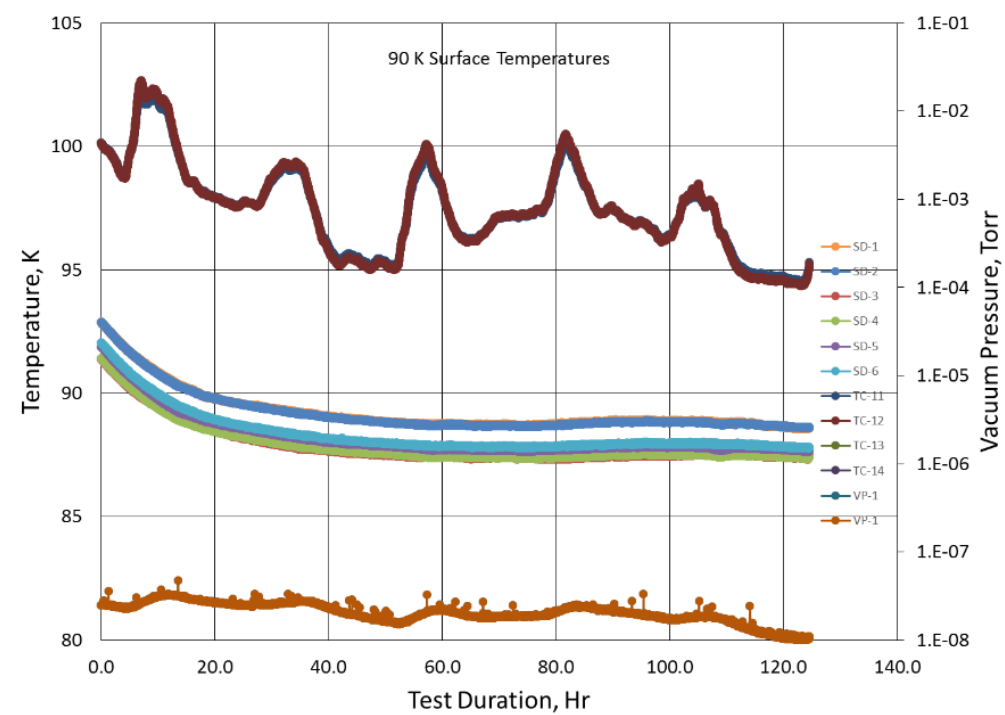

Figure 10 Warm boundary temperatures for double aluminized Mylar testing.

From the data shown in Table 3, it is evident that the energy passing through the foil was much greater than passing through the DAM at both temperatures tested. It is also interesting that both systems show an increase in effective 
emissivity between the two cases. This suggests that the increase in emissivity that was previously attributed to transmission is not restricted to DAM alone, but may also be apparent in foils [9].

An alternative model assuming a constant parasitic heat load on the calorimeter was suggested by Yongquist [11]. This model assumed that the effective emmisivity is constant between temperatures with a constant parasitic heat load. In order to linearize the model, the parasitic heat flux through the foil was $82.2 \mathrm{~mW} / \mathrm{m}^{2}$ and through the DAM was $37.4 \mathrm{~mW} / \mathrm{m}^{2}$. The effective emissivities were 0.0033 for the foil and 0.0026 for the DAM. Again the DAM had a lower effective emissivity than the foil.

As stated at the start any transmissivity in the DAM vis-a-vis the aluminum foil should show as a higher heat load in a low boundary temperature test. However our test results consistently show a lower heat load for DAM than aluminum foil. Based on this data it is unlikely that any increase heat load at low temperatures can be attributed to transmission through DAM.

\section{Summary}

A new calorimeter for testing cryogenic insulation has been successfully constructed and is now operational. Work on single layer transmissivity has indicated that the long held speculation that DAM might be transparent at low temperatures may be incorrect. Direct transmissivity measurements of DAM samples are under way as well, to further validate these findings. Further testing with full multilayer insulation blankets and different seaming techniques is planned in the near future.

\section{References}

[1] J. E. Fesmire, S. D. Augustynowicz, K. W. Heckle and B. N. Scholtens, Equipment and Methods for Cryogenic Thermal Insulation Testing, Anchorage, AK: Cryogenic Engineering Conference, September 2003.

[2] W. L. Johnson, Thermal Performance of Cryogenic Multilayer Insulation at Various Layer Spacings, Master's Thesis: University of Central Florida, 2010.

[3] D. Celik, R. Klimas, S. Van Sciver and J. Zia, "An Apparatus to Measure the Apparent Thermal Conductivity of Multilayer Insulation (MLI)," in Advances in Cryogenic Engineering, Vol 57B, Melville, NY, American Institute of Physics, 2012, pp. 1313-1318.

[4] J. A. Hurd, Multilayer Insulation Test at Variable Boundary Temperatures, Tallahassee, FL: Florida State University, 2013.

[5] T. Funke and C. Haberstroh, "A Calorimeter for Measurements of Multilayer Insulation at Variable Cold Temperature," in 25th International Cryogenic Engineering Conference, Enschede, The Netherlands, 2014.

[6] American Society for Testing and Materials, "ASTM E-408-13 Standard Test Methods for Total Normal Emittance of Surfaces Using Inspection-Meter Techniques," ASTM International, West Conshohocken, PA, 2013.

[7] W. L. Johnson, J. G. Valenzuela, J. R. Feller, and D. W. Plachta “Tank Applied Testing of Load-Bearing Multilayer Insulation (LB-MLI) AIAA 2014-3581 AIAA/ASME/SAE/ASEE 50th Joint Propulsion Conference, Cleveland, OH, 2014.

[8] W. N. Borowski, T. H. Nicol and C. J. Schoo "Thermal Performance of Various Multilayer Insulation Systems Below 80K” Supercollider 4 J. Nante, ed. Springer, NY pg. 235-247.

[9] I. E. Spradley, T. C. Nast, D. J. Frank "Experimental Studies of MLI Systems at Very Low Boundary Temperatures," Advances in Cryogenic Engineering, Vol. 35 R. W. Fast ed., Plenum Press , NY, 1990, pg 477-486.

[10] J. Tuttle, M. DiPirro, E. Canavan and T. Hait “Thermal Properties of Double Aluminized Kapton at Low Temperatures" AIP conference Proceedings 986(1), pg. 34-41

[11] Personal communication with Robert Youngquist, NASA Kenedy Space Flight Center 